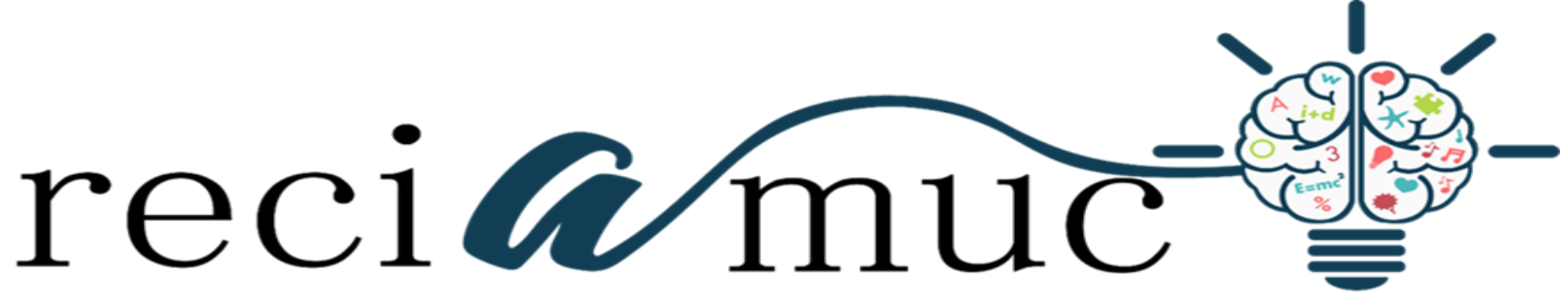

Revista científica de investigación actualización del mundo de las ciencias

María Belén Gárate Campoverde a ; Cástulo Leonel Moreira Moreira ${ }^{\text {b; Juan }}$

Carlos Zambrano Basurto c; María José Saltos Loor ${ }^{\text {d; }}$ Stephane Paolo Guerrero

Zambrano $^{\mathrm{e}}$; Rómulo Argenis Muñoz Moreira ${ }^{\mathrm{f}}$

Cuidados de las pacientes sometidas a cirugías de cáncer uterino

Care of patients subjected to uterine cancer surgeries

Revista Científica de Investigación actualización del mundo de las Ciencias. Vol.

3 núm., 3, julio, ISSN: 2588-0748, 2018, pp. 1323-1346

DOI: $10.26820 /$ reciamuc/3.(3).julio.2019.1323-1346

URL: $\underline{\text { http://reciamuc.com/index.php/RECIAMUC/article/view/392 }}$

Código UNESCO: 3205 Medicina Interna

Tipo de Investigación: Artículo de Investigación

(C) RECIAMUC; Editorial Saberes del Conocimiento, 2019

Recibido: 28/04/2019 Aceptado: 19/05/2019 Publicado: 01/07/2019

Correspondencia: belen_garate@hotmail.com

a. Médica; Investigador Independiente; Guayaquil - Ecuador; belen_garate@ hotmail.com

b. Médico General Asistencial Cirugía General; Hospital Dr. Verdi Cevallos Balda; Portoviejo - Ecuador; castuloleonel@hotmail.com

c. Médico General Asistencial Cirugía General; Hospital Dr. Verdi Cevallos Balda; Portoviejo - Ecuador; juankzb_89@hotmail.com

d. Médico General Asistencial Anestesiología; Hospital Dr. Verdi Cevallos Balda; Portoviejo - Ecuador; maj_o_sl@hotmail.com

e. Médico General Asistencial Cirugía General; Hospital Dr. Verdi Cevallos Balda; Portoviejo - Ecuador.

f. Médico Residente en funciones hospitalarias; Hospital Básico San Andrés de Flavio; Portoviejo - Ecuador; romuloargenis89@gmail.com 


\section{Cuidados de las pacientes sometidas a cirugías de cáncer uterino}

Vol. 3, núm. 1., (2019)

María Belén Gárate Campoverde; Cástulo Leonel Moreira Moreira; Juan Carlos Zambrano

Basurto; María José Saltos Loor; Stephane Paolo Guerrero Zambrano; Rómulo Argenis Muñoz Moreira

\section{RESUMEN}

Desde hace muchos años han venido surgiendo una serie de enfermedades a nivel Mundial, las cuales han llevado al desarrollo de múltiples investigaciones científicas dirigidas a intentar erradicarlas, o por lo menos mejorar los tratamientos en torno a las mismas. He aquí, que uno de los descubrimientos más importantes, son los correspondientes a la investigación oncológica, donde se han demostrado evidencias científicas, provenientes de estudios virológicos, moleculares, clínicos y epidemiológicos que han permitido demostrar y describir de forma inequívoca que el cáncer de cuello de útero es sin duda un importante problema de salud a nivel mundial y representa una de las principales causas de muerte en la mujer. Esta anomalía oncológica, se ha convertido en la primera causa de muerte en el grupo etáreo entre los 20 a 44 años y la sexta en el grupo de 45 a 64 años, teniendo un importante impacto social y económico, puesto que afecta esencialmente a mujeres jóvenes y en edad reproductiva. La calidad de vida y experiencia de estas pacientes, es totalmente subjetiva, y está fuertemente relacionada con el grado de satisfacción que tiene la persona con su estado físico, emocional, con su vida familiar, amorosa, y social. Datos epidemiológicos resaltan que entre las cirugías más comunes realizadas a pacientes con esta patología se encuentra la histerectomía radical. Otro de los procesos lo representa la Cervicectomía y la disección de ganglios linfáticos pélvicos, su operación es realizada a través de la vagina o el abdomen, y a veces se hace mediante laparoscopia. Entre los cuidados a los que deben someterse las personas afectadas se encuentran los tratamientos de radioterapia y los de evisceración pélvica. Asimismo, se encuentran los paliativos, destinados a aquellas personas en las que el cáncer es terminal y busca mejorar el proceso terminal del enfermo.

Palabras Claves: Cirugías de cáncer Uterino; Histerectomía; Cervicectomía; Disección de Ganglios Pélvicos; Cuidados Paliativos. 


\section{Cuidados de las pacientes sometidas a cirugías de cáncer uterino}

Vol. 3, núm. 1., (2019)

María Belén Gárate Campoverde; Cástulo Leonel Moreira Moreira; Juan Carlos Zambrano

Basurto; María José Saltos Loor; Stephane Paolo Guerrero Zambrano; Rómulo Argenis Muñoz

Moreira

\section{ABSTRACT}

A number of diseases have been emerging worldwide for many years, which have led to the development of multiple scientific research aimed at eradicating them, or at least improving the treatments around them. Here, one of the most important discoveries, are those corresponding to oncological research, where scientific evidence has been demonstrated, coming from virological, molecular, clinical and epidemiological studies that have allowed to unequivocally demonstrate and describe that neck cancer Uterus is undoubtedly a major health problem worldwide and represents one of the leading causes of death in women. This oncological anomaly has become the first cause of death in the age group between 20 and 44 years old and the sixth in the group of 45 to 64 years, having an important social and economic impact, since it essentially affects young women and in reproductive age. The quality of life and experience of these patients, is totally subjective, and is strongly related to the degree of satisfaction that the person has with their physical, emotional, family, love, and social life. Epidemiological data highlight that between The most common surgeries performed on patients with this pathology is radical hysterectomy. Another process is represented by Cervicectomy and pelvic lymph node dissection, its operation is performed through the vagina or abdomen, and sometimes it is done by laparoscopy. Among the cares to which the affected people must undergo are radiotherapy and pelvic evisceration treatments. Also, there are the palliative ones, destined to those people in whom the cancer is terminal and seeks to improve the terminal process of the patient.

Key Words: Uterine cancer surgeries; Hysterectomy; Cervicectomy; Pelvic Gland Dissection; Palliative Care. 


\section{Cuidados de las pacientes sometidas a cirugías de cáncer uterino}

Vol. 3, núm. 1., (2019)

María Belén Gárate Campoverde; Cástulo Leonel Moreira Moreira; Juan Carlos Zambrano

Basurto; María José Saltos Loor; Stephane Paolo Guerrero Zambrano; Rómulo Argenis Muñoz Moreira

\section{Introducción.}

El cáncer de cuello de útero representa el resultado final de una enfermedad venérea no resuelta. $\mathrm{Su}$ implicación causal en el cáncer de cuello de útero, ha ido revolucionando las opciones preventivas de que se disponía hasta ahora, ofreciendo una oportunidad única para introducir nuevas estrategias de prevención primaria y secundaria basadas en el VPH. Según la Guía Clínica de cáncer cervicouterino (CACU) (OMS, 2017), esta es una alteración celular que se origina en el epitelio del cuello del útero y que inicialmente se manifiesta como lesiones precursoras de lenta y progresiva evolución, las cuales se suceden en etapas de displasia leve, moderada y severa, según sea el nivel de invasión del tejido afectado.

En este contexto, se describe que esta enfermedad puede evolucionar a cáncer in situ (cuando están circunscritas a la superficie epitelial) y/o cáncer invasor cuando el compromiso traspasa la membrana basal. Generalmente este tipo de cáncer se disemina localmente, es decir, penetra solo estructuras adyacentes como parámetros, vagina, cuerpo del útero, vejiga, uréteres y recto, habitualmente sin metástasis a distancia por vía hematógena. Esta última solo se presenta como una manifestación tardía de la enfermedad, afectando a órganos como pulmones, hígado, y hueso principalmente. (Weiss, 1997).

El Cáncer Cervicouterino es un enemigo tan silencioso para la mujer, que éstas a menudo no presentan sintomatología en las etapas iniciales de la enfermedad, por lo que muchas veces no se detecta sino hasta que se hace severo y su reversibilidad es casi nula. (Henalt, 2016). En efecto, el cáncer de cuello uterino, CACU es sin duda un importante problema de salud a nivel mundial, pues a pesar de ser uno de los tipos de cáncer más fáciles de detectar y prevenir debido a que su desarrollo es gradual, se ha convertido en una de las principales causas de muerte en la mujer cobrando cada año la vida de 231.000 mujeres en el mundo, perteneciendo el $80 \%$ de éstas a países subdesarrollados (Henalt, 2016).

El factor de riesgo más frecuente para este tipo de cáncer es la exposición o contagio a ciertas variedades de Papiloma virus Humano (HPV), el cual constituye una enfermedad de transmisión sexual (ETS) asintomática en la mayoría de los casos, por lo que no es una sorpresa que puedan 


\section{Cuidados de las pacientes sometidas a cirugías de cáncer uterino}

Vol. 3, núm. 1., (2019)

María Belén Gárate Campoverde; Cástulo Leonel Moreira Moreira; Juan Carlos Zambrano Basurto; María José Saltos Loor; Stephane Paolo Guerrero Zambrano; Rómulo Argenis Muñoz

Moreira

pasar largos periodos de tiempo antes de que su presencia sea detectada. (Henalt Ob cit). La Revista Chilena de Obstetricia y Ginecología menciona la existencia de más de 70 tipos de HPV, cada uno con una preferencia particular por algún sitio anatómico del cuerpo, siendo los tipos 16, 18 y 31, los que guardan estrecha relación con la aparición y desarrollo del CACU en la mujer.

Esto no es un tema menor si consideramos que la infección por HPV es considerada la enfermedad de transmisión sexual más común del mundo, afectando al 50\% y 80\% de las mujeres al menos una vez en sus vidas, tal como nos muestra la American Journal of Medicine (2017). Sin embargo, es importante aclarar que existen otros factores de riesgo ligados al desarrollo del CACU en la mujer. En este aspecto, la Organización Panamericana de la Salud (2017) destaca como los más frecuentes (especialmente en los países en desarrollo), el fuerte desapego de las mujeres a realizarse el examen para la detección oportuna del cáncer y prevención de éste, inicio sexual a temprana edad y parejas sexuales múltiples o que estén contagiadas.

Esto último, conlleva un doble riesgo si se considera la posibilidad latente de contagio con el Virus de Inmunodeficiencia Humana (VIH), que al debilitar el sistema inmunológico de la mujer la hace más vulnerable a contraer la infección. Asimismo, el tener una historia familiar donde existan casos anteriores de CACU, pertenecer a un grupo etáreo de riego (30 - 60 años), y adoptar estilos de vida poco saludable como fumar, también denotan un aumento en la posibilidad de desarrollar la enfermedad. 


\section{Cuidados de las pacientes sometidas a cirugías de cáncer uterino}

Vol. 3, núm. 1., (2019)

María Belén Gárate Campoverde; Cástulo Leonel Moreira Moreira; Juan Carlos Zambrano Basurto; María José Saltos Loor; Stephane Paolo Guerrero Zambrano; Rómulo Argenis Muñoz Moreira

\section{Imagen $\mathbf{N}^{\circ}$ 1. Zonas afectadas con el Cáncer Uterino}

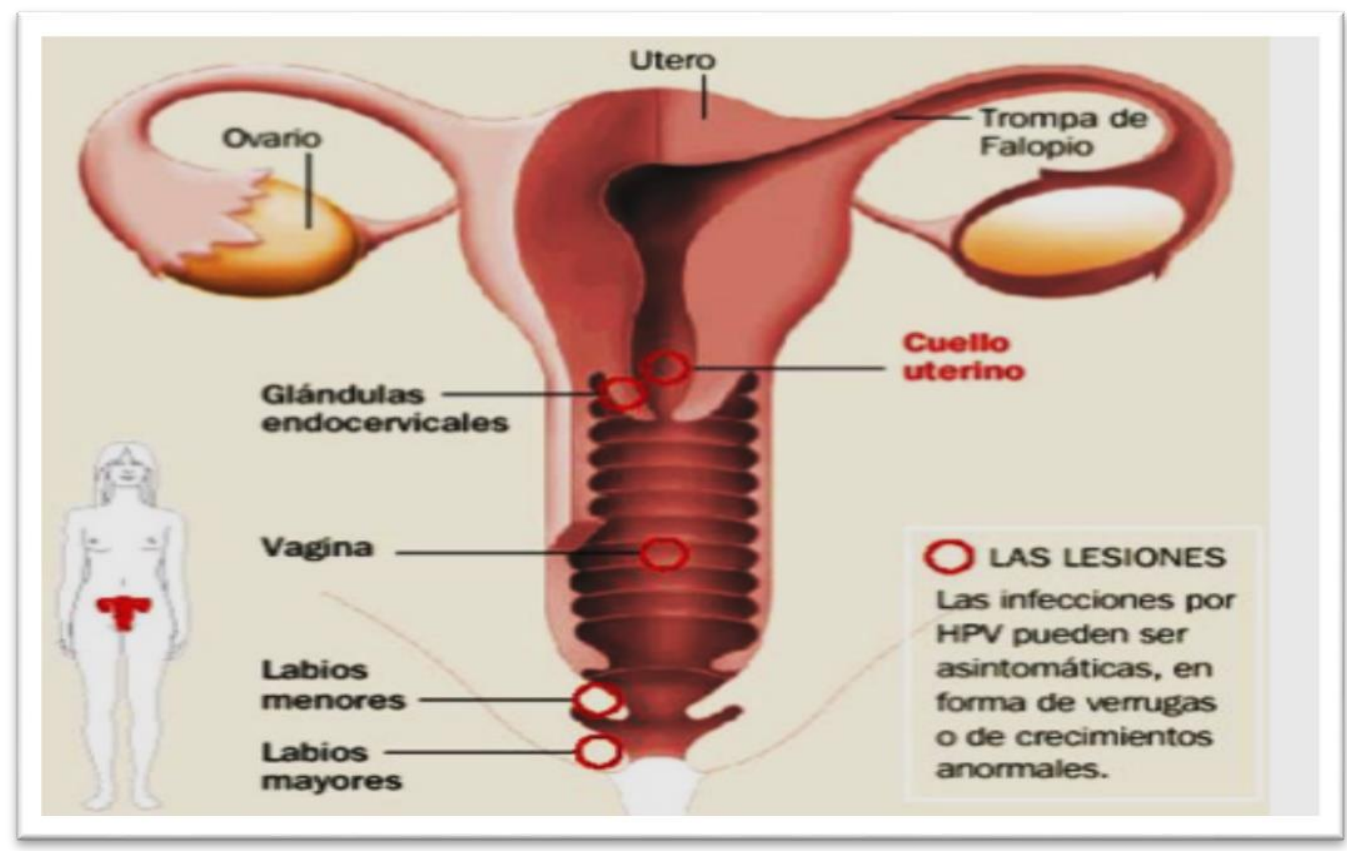

Fuente: (Henalt 2016).

\section{Tipo de Investigación.}

Dentro de toda práctica investigativa, se precisan acciones de carácter metodológico mediante las cuales, se logra conocer y proyectar los eventos posibles que la determinan, así como las características que hacen del acto científico un proceso interactivo ajustado a una realidad posible de ser interpretada. En este sentido, se puede decir, que la presente investigación corresponde al tipo documental, definido por (Dávila, 2012), "se ocupa del estudio de problemas planteados a nivel teórico, la información requerida para abordarlos se encuentra básicamente en materiales impresos, audiovisuales y /o electrónicos”. (p.41).

En consideración a esta definición, la orientación metodológica permitió la oportunidad de cumplir con una serie de actividades inherentes a la revisión y lectura de diversos documentos donde se encontraron ideas explicitas relacionadas con los tópicos encargados de identificar a cada característica insertada en el estudio. Por lo tanto, se realizaron continuas interpretaciones con el claro propósito de revisar aquellas apreciaciones o investigaciones propuestas por 


\section{Cuidados de las pacientes sometidas a cirugías de cáncer uterino}

Vol. 3, núm. 1., (2019)

María Belén Gárate Campoverde; Cástulo Leonel Moreira Moreira; Juan Carlos Zambrano

Basurto; María José Saltos Loor; Stephane Paolo Guerrero Zambrano; Rómulo Argenis Muñoz

Moreira

diferentes investigadores, para luego dar la respectiva argumentación a los planteamientos, en función a las necesidades encontradas en la indagación.

\section{Fuentes Documentales.}

El análisis correspondiente a las características que predomina en el tema seleccionado, llevan a incluir diferentes fuentes documentales encargadas de darle el respectivo apoyo y en ese sentido cumplir con la valoración de los hechos a fin de generar nuevos criterios que sirven de referencia a otros procesos investigativos. Para (Arias, 2010), las fuentes documentales incorporadas en la investigación documental o bibliográfica, "representa la suma de materiales sistemáticos que son revisados en forma rigurosa y profunda para llegar a un análisis del fenómeno". (p.41). Por lo tanto, se procedió a cumplir con la realización de una lectura previa determinada por encontrar aquellos aspectos estrechamente vinculados con la "Prevención y cuidados ginecológicos de la mujer en edad Fértil" para luego explicar mediante un desarrollo las respectivas apreciaciones generales de importancia.

\section{Técnicas para la Recolección de la Información.}

La conducción de la investigación para ser realizada en función a las particularidades que determinan a los estudios documentales, tiene como fin el desarrollo de un conjunto de acciones encargadas de llevar a la selección de técnicas estrechamente vinculadas con las características del estudio. En tal sentido, (Arias Ob cit) refiere, que es "una técnica particular para aportar ayuda a los procedimientos de selección de las ideas primarias y secundarias". (p. 71).

Por ello, se procedió a la utilización del subrayado, resúmenes, fichaje, como parte básica para la revisión y selección de los documentos que presentan el contenido teórico. Es decir, que mediante su aplicación de estas técnicas se pudo llegar a recoger informaciones en cuanto a la revisión bibliográfica de los diversos elementos encargados de orientar el proceso de investigación. Tal como lo expresa, (Arias Ob cit) "las técnicas documentales proporcionan las herramientas esenciales y determinantes para responder a los objetivos formulados y llegar a resultados efectivos" (p. 58). Es decir, para responder con eficiencia a las necesidades 


\section{Cuidados de las pacientes sometidas a cirugías de cáncer uterino}

Vol. 3, núm. 1., (2019)

María Belén Gárate Campoverde; Cástulo Leonel Moreira Moreira; Juan Carlos Zambrano

Basurto; María José Saltos Loor; Stephane Paolo Guerrero Zambrano; Rómulo Argenis Muñoz Moreira

investigativas, se introdujeron como técnica de recolección el método inductivo, que hizo posible llevar a cabo una valoración de los hechos de forma particular para llegar a la explicación desde una visión general.

Asimismo, se emplearon las técnicas de análisis de información para la realización de la investigación que fue ejecutada bajo la dinámica de aplicar diversos elementos encargados de determinar el camino a recorrer por el estudio, según, (Arias, Ob cit) las técnicas de procesamiento de datos en los estudios documentales "son las encargadas de ofrecer al investigador la visión o pasos que debe cumplir durante su ejercicio, cada una de ellas debe estar en correspondencia con el nivel a emplear" (p. 123). Esto indica, que para llevar a cabo el procesamiento de los datos obtenidos, es necesario establecer las técnicas que serán seleccionadas, destacándose en este caso, de manera particular: fichas de resumen, textual, registros descriptivos entre otros, los mismos se deben ajustar al nivel que ha sido seleccionado.

\section{Resultados.}

\section{Cáncer de Cuello Uterino}

El cáncer de cuello uterino es la quinta neoplasia en el mundo según la OMS y el segundo tipo de cáncer más común en mujeres, el incremento mundial de las mujeres con cáncer de útero desde hace 10 años ha sido inminente. Antes se diagnosticaban 500 mil casos al año ahora son 700 mil casos al año. Si hay presencia de células cancerosas en la muestra, el médico determinará cual es el procedimiento más adecuado para combatir el CACU.

De manera general, el cáncer de cuello uterino se ve asociado a la histerectomía radical; procedimiento éste que amerita ser aplicado cuando se extrae el cuello uterino, el útero y parte de la vagina, además de los ganglios linfáticos presentes en la región afectada. Según la forma de extracción del útero se llamará histerectomía vaginal (cuando este se extrae vía vaginal), o abdominal (si es extraído a través del abdomen). El tratamiento de la enfermedad invasora temprana se realiza principalmente mediante cirugía, en etapas más avanzadas, las opciones son 


\section{Cuidados de las pacientes sometidas a cirugías de cáncer uterino}

Vol. 3, núm. 1., (2019)

María Belén Gárate Campoverde; Cástulo Leonel Moreira Moreira; Juan Carlos Zambrano

Basurto; María José Saltos Loor; Stephane Paolo Guerrero Zambrano; Rómulo Argenis Muñoz

Moreira

cirugía o radioterapia (Weiss, 1997). Finalmente, las opciones que puede utilizar se basan en de 3 tipos de tratamiento:

La Cirugía, es un procedimiento quirúrgico (operación) en la cual se extrae el útero, y cuello uterino además del cáncer. Esta terapia oncológica depende de la etapa y volumen de la lesión primaria, la edad de la paciente y los problemas médicos concurrentes que pudiese presentar. La cirugía consiste en histerectomía de diversos grados de radicalidad, siempre dependientes de los niveles y estructuras invadidas y con uso selectivo de linfa denectomía.

La Quimioterapia, es un tratamiento sistémico que consiste en el uso de agentes químicos o medicamentos por vía intravenosa o arterial para destruir células malignas. La quimioterapia se usa frecuentemente con cirugía o radioterapia para tratar el cáncer. Algunos tratamientos de quimioterapia tienen etapas diferentes:

Inducción, que es un tratamiento intensivo con la intención de causar remisión completa.

Mantenimiento, donde los medicamentos se administran después de la etapa inicial de inducción para mantener la remisión del cáncer. (Weiss, 1997).

Según su fin terapéutico, se puede clasificar los tipos de quimioterapia como:

A. "Quimioterapia concurrente con radioterapia" la cual se usa para enfermedad localmente avanzada y es administrada al mismo tiempo que la radioterapia como tratamiento neo adyuvante de la cirugía o como único tratamiento (definitivo).

B. "Quimioterapia para enfermedad metastásica o recaída" la cual se usa en aquellas mujeres que a pesar de haber sido anteriormente tratadas muestran signos de nuevas infiltraciones de células cancerígenas en algún lugar de su organismo.

C. "Quimioterapia neo adyuvante" cuando es administrada previo al tratamiento definitivo, generalmente la cirugía. (Weiss, 1997)

La elección de cada una de estas terapias generalmente se encuentra limitada al tratamiento recibido por cada una de las pacientes. Esta aserción se sustenta en el pensamiento de que la 


\section{Cuidados de las pacientes sometidas a cirugías de cáncer uterino}

Vol. 3, núm. 1., (2019)

María Belén Gárate Campoverde; Cástulo Leonel Moreira Moreira; Juan Carlos Zambrano

Basurto; María José Saltos Loor; Stephane Paolo Guerrero Zambrano; Rómulo Argenis Muñoz Moreira

actividad del fármaco quimio terápico se afecta por la vascularidad tumoral deficiente originada por tratamientos quirúrgicos o radioterápicos anteriores que la mujer pudiese haber recibido, además, las recidivas pélvicas de la enfermedad ocasionan infección e inanición, lo que merma el estado de funcionamiento de la paciente y su tolerancia al tratamiento (Weiss, 1997)

Hay muchos factores que afectan el pronóstico del cáncer cervical, a saber:

- El tipo de cáncer

- La etapa de la enfermedad

- La edad y condición física general de la mujer

Las afecciones precancerosas son completamente curables cuando se les hace un seguimiento y tratamiento apropiado. La probabilidad de estar vivo a los 5 años responde a la (tasa de supervivencia a 5 años) para el cáncer que se ha diseminado al interior de las paredes del cuello uterino, pero no por fuera del área de éste, es del $92 \%$.

Sin embargo, la tasa de supervivencia a 5 años disminuye regularmente a medida que el cáncer se disemina a otras áreas.

\section{Complicaciones:}

- Algunos tipos de cáncer de cuello uterino no responden bien al tratamiento.

- El cáncer puede retornar (reaparecer) después del tratamiento.

- Las mujeres que reciben tratamiento para salvar el útero tienen un alto riesgo de retorno (reaparición) del cáncer.

- La cirugía y la radiación pueden causar problemas con la función sexual, intestinal y vesical.

Estudios oncológicos han demostrado la existencia de evidencias provenientes de investigaciones virológicas, moleculares, clínicas y epidemiológicas que han permitido demostrar y describir de forma inequívoca que el cáncer de cuello de útero es, en realidad, una secuela a largo plazo de 


\section{Cuidados de las pacientes sometidas a cirugías de cáncer uterino}

Vol. 3, núm. 1., (2019)

María Belén Gárate Campoverde; Cástulo Leonel Moreira Moreira; Juan Carlos Zambrano Basurto; María José Saltos Loor; Stephane Paolo Guerrero Zambrano; Rómulo Argenis Muñoz

Moreira

una infección persistente por ciertos genotipos de $\mathrm{VPH}$, un virus de transmisión primordialmente sexual.

\section{Imagen $\mathbf{N}^{\circ}$ 2. Cáncer de Cuello Uterino (CCU)}

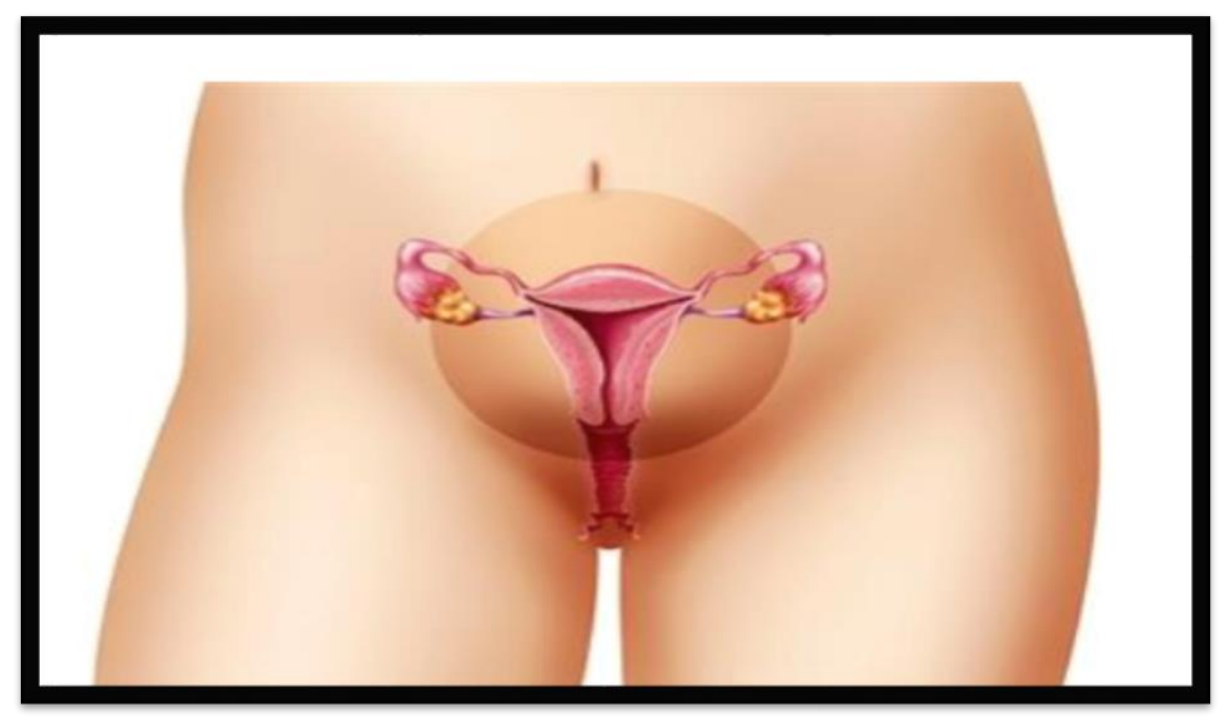

Fuente: (Weiss 1997).

\section{Diagnóstico}

Existen múltiples estudios que pueden ser realizados con el fin de determinar si una paciente padece o no, de cáncer cuello uterino; entre ellos se mencionan los siguientes:

Las Citologías Vaginales: detectan los pre cánceres y el cáncer, pero no ofrecen el diagnóstico final. Si se encuentran cambios anormales, generalmente se examina el cuello uterino bajo aumento o ampliación microscópica, lo cual se denomina Colposcopia. Si se observa alguna lesión sospechosa debe realizarse una biopsia o curetaje endo cervical, de ser el caso. Este procedimiento consiste en extraer fragmentos de tejido en forma quirúrgica (biopsia) y se envían al laboratorio para su análisis. Los diagnósticos diferenciales incluyen: - Mioma Abortado Pólipo endocervical - Tejido de granulación. 


\section{Cuidados de las pacientes sometidas a cirugías de cáncer uterino}

Vol. 3, núm. 1., (2019)

María Belén Gárate Campoverde; Cástulo Leonel Moreira Moreira; Juan Carlos Zambrano

Basurto; María José Saltos Loor; Stephane Paolo Guerrero Zambrano; Rómulo Argenis Muñoz Moreira

PAP: Es una prueba de detección para cáncer de cuello uterino. Las células tomadas por raspado de la abertura del cuello uterino se examinan bajo un microscopio. El cuello uterino es la parte más baja del útero (matriz) que desemboca en la parte superior de la vagina. El médico introduce un espéculo. Esto le permite observar el interior de la vagina y el cuello uterino. Se raspan células suavemente del área del cuello uterino y la muestra se envía a un laboratorio para su análisis

Biopsia: Una biopsia de cuello uterino es un procedimiento que se realiza para extraer tejido del cuello uterino con el fin de detectar condiciones anormales o precancerosas, o cáncer de cuello uterino. El cuello uterino es la parte inferior y estrecha del útero ubicada entre la vejiga y el recto. Forma un canal que desemboca en la vagina, la que a su vez conduce al exterior del cuerpo. B

\section{Imagen $\mathbf{N}^{\circ}$ 3. Examen de Biopsia}

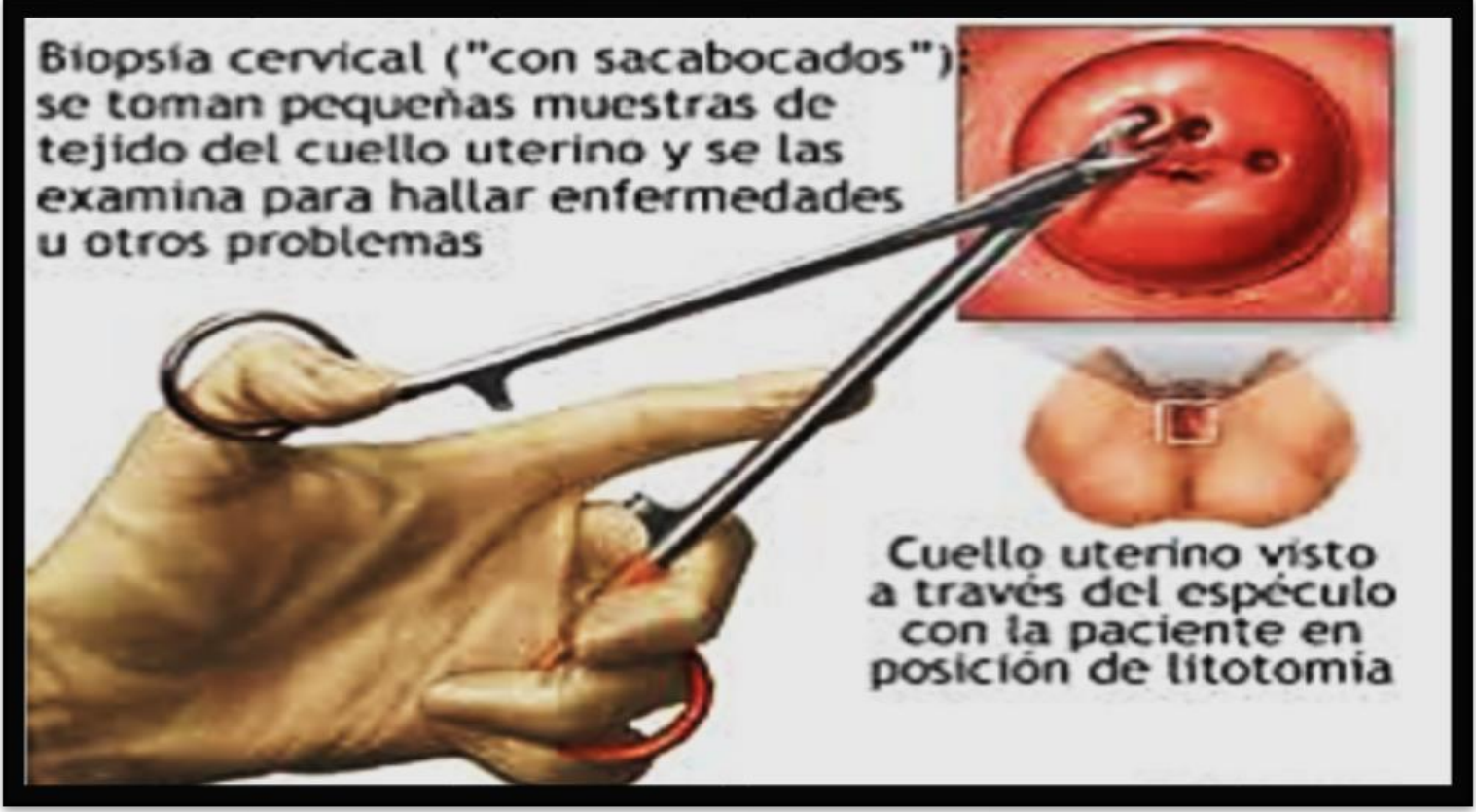

Fuente: (Weiss 1997).

CONO LEEP: Es un tratamiento para las células anormales del cuello del útero. El LEEP, forma abreviada del inglés que significa procedimiento de escisión electro quirúrgica con asa, elimina el tejido anormal usando un asa de alambre fino que transmite corriente eléctrica. Se puede hacer 


\section{Cuidados de las pacientes sometidas a cirugías de cáncer uterino}

Vol. 3, núm. 1., (2019)

María Belén Gárate Campoverde; Cástulo Leonel Moreira Moreira; Juan Carlos Zambrano

Basurto; María José Saltos Loor; Stephane Paolo Guerrero Zambrano; Rómulo Argenis Muñoz

Moreira

cuando se han identificado células anormales mediante una prueba de Papanicolaou, una colposcopía o una biopsia

CONO FRÍO: La biopsia de cono frío es un procedimiento quirúrgico que se lleva a cabo bajo anestesia general y se recomienda cuando se presentan cambios precancerosos en el cuello uterino.

\section{Cirugías de Cuello Uterino}

Muchas mujeres con cáncer de cuello uterino tendrán que someterse a algún tipo de cirugía. La cirugía se puede usar para:

- Ayudar a diagnosticar el cáncer de cuello uterino

- Ayudar a determinar cuán lejos se ha propagado el cáncer

- Ayudar a tratar el cáncer (especialmente para los cánceres en etapas más tempranas)

Se pueden usar varios tipos de 'cirugía' para ayudar a tratar el cáncer de cuello uterino, aunque algunos de estos destruyen el tejido cervical (con frío o con láser) en lugar de extraerlo.

\section{Histerectomía Simple (Total)}

Esta cirugía se hace para extirpar el útero (tanto el cuerpo del útero como el cuello uterino), pero no las estructuras próximas al útero (parametrio y ligamentos uterosacros). Ni la vagina ni los ganglios linfáticos de la pelvis se extirpan. Los ovarios y las trompas de Falopio usualmente se dejan en su lugar a menos que haya otra razón para extirparlos.

- Existen diferentes maneras de hacer una histerectomía:

- Histerectomía abdominal: el útero se extirpa a través de una pequeña incisión quirúrgica que se hace en la parte frontal del abdomen.

- Histerectomía vaginal: el útero se extirpa a través de la vagina.

- Histerectomía laparoscópica: el útero se extrae usando laparoscopia (a veces llamada “cirugía mínimamente invasiva”). Un tubo delgado con una cámara de video diminuta en 


\section{Cuidados de las pacientes sometidas a cirugías de cáncer uterino}

Vol. 3, núm. 1., (2019)

María Belén Gárate Campoverde; Cástulo Leonel Moreira Moreira; Juan Carlos Zambrano Basurto; María José Saltos Loor; Stephane Paolo Guerrero Zambrano; Rómulo Argenis Muñoz Moreira

el extremo (el laparoscopio) se inserta en una o más pequeñas incisiones quirúrgicas para ver el interior del abdomen y la pelvis. Instrumentos pequeños se pueden controlar a través del tubo, permitiendo que el cirujano realice cortes y extraiga tejido a través de los tubos sin hacer una incisión grande en el abdomen.

- Histerectomía vaginal asistida por laparoscopia: El útero, los ovarios y las trompas de Falopio se extirpan a través de una incisión vaginal utilizando el laparoscopio, lo que facilita la labor dl médico.

- Cirugía asistida por robot: en este método, la laparoscopia se realiza con herramientas especiales conectadas a brazos robóticos que son controlados por el médico para ayudar a realizar una cirugía precisa.

Es importante resaltar que para todas estas operaciones se usa anestesia general o epidural (regional), entre sus efectos se deben mencionar:

Para una histerectomía vaginal o laparoscópica, la hospitalización es usualmente de uno a dos días, y la recuperación demora de dos a tres semanas. Para una histerectomía abdominal se requiere usualmente de 3 a 5 días de hospitalización, y la recuperación completa toma alrededor de 4 a 6 semanas.

Posibles efectos secundarios: Cualquier tipo de histerectomía causa esterilidad (incapacidad para tener hijos). Normalmente no hay complicaciones, pero pudiera presentarse sangrado excesivo, infección de la herida o daños a los sistemas urinario o intestinal.

La histerectomía no cambia la capacidad en una mujer de sentir placer sexual. Una mujer no necesita un útero o cuello uterino para alcanzar un orgasmo. El área alrededor del clítoris y la membrana que recubre la vagina permanecen tan sensitivas como antes de una histerectomía. Para más información sobre cómo manejar los efectos secundarios sexuales del tratamiento del cáncer de cuello uterino, lea El sexo y la mujer con cáncer. 


\section{Cuidados de las pacientes sometidas a cirugías de cáncer uterino}

Vol. 3, núm. 1., (2019)

María Belén Gárate Campoverde; Cástulo Leonel Moreira Moreira; Juan Carlos Zambrano Basurto; María José Saltos Loor; Stephane Paolo Guerrero Zambrano; Rómulo Argenis Muñoz

Moreira

\section{Histerectomía Radical}

Para esta operación, el cirujano extirpa el útero junto con los tejidos contiguos al útero (el parametrio y los ligamentos uterosacros), así como la parte superior (alrededor de una pulgada) de la vagina adyacente al cuello uterino. Los ovarios y las trompas de Falopio no se extirpan a no ser que haya otra razón médica para hacerlo. En la histerectomía radical se extirpa más tejido que en la histerectomía simple, por lo que la estadía en el hospital puede ser más prolongada.

\section{Imagen $\mathbf{N}^{\circ}$ 4. Histerectomía}

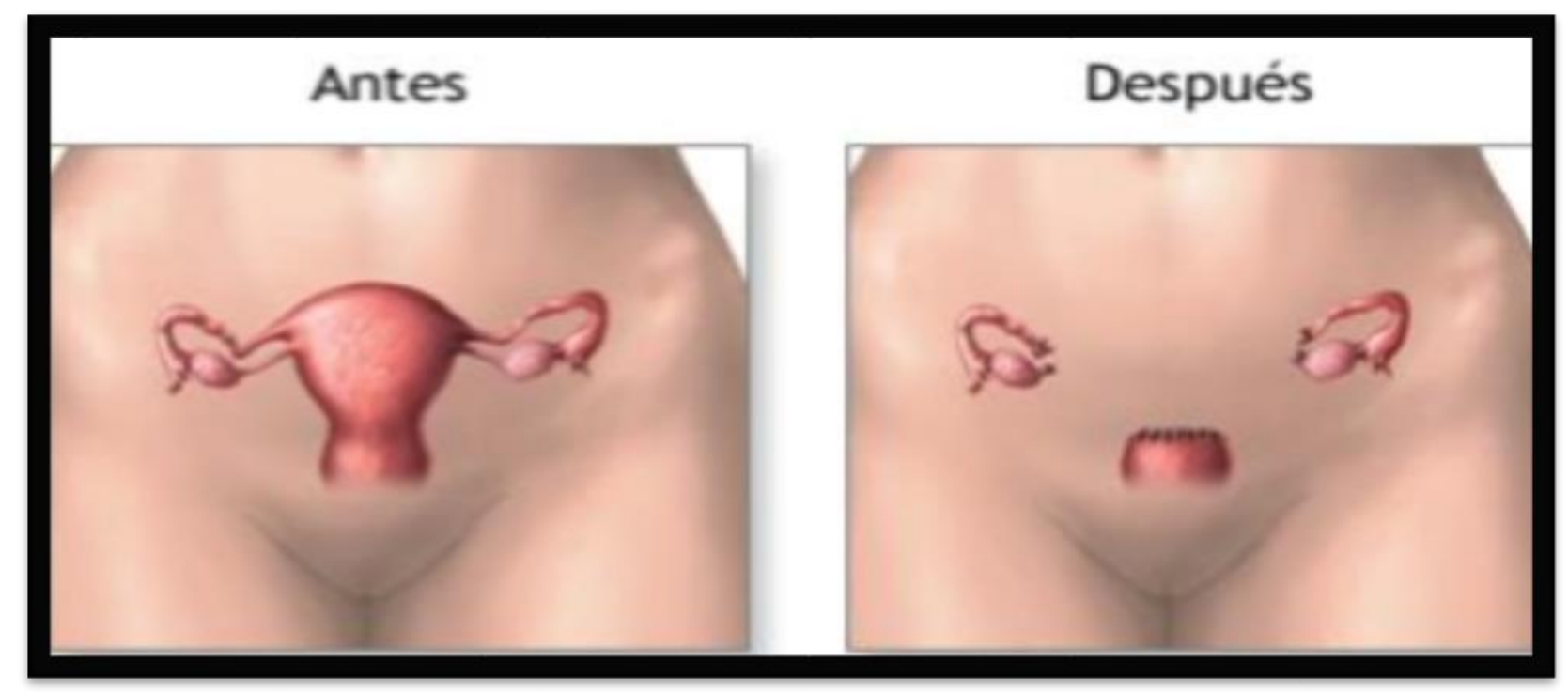

Fuente: (Weiss 1997)

Esta cirugía generalmente se hace a través de una incisión grande en el abdomen (conocida como cirugía abierta). A menudo, también se extirpan algunos ganglios linfáticos pélvicos. (Este procedimiento, conocido como disección de ganglios linfáticos, se discute más adelante en esta sección).

También se puede hacer una histerectomía radical mediante laparoscopia (cirugía mínimamente invasiva). (Véase la sección 'Histerectomía Simple’ para una descripción de la laparoscopia). 


\section{Cuidados de las pacientes sometidas a cirugías de cáncer uterino}

Vol. 3, núm. 1., (2019)

María Belén Gárate Campoverde; Cástulo Leonel Moreira Moreira; Juan Carlos Zambrano

Basurto; María José Saltos Loor; Stephane Paolo Guerrero Zambrano; Rómulo Argenis Muñoz Moreira

La histerectomía vaginal radical asistida por laparoscopia es un método quirúrgico que combina una histerectomía vaginal radical con una disección laparoscópica de ganglios linfáticos pélvicos.

El laparoscopio también puede facilitar la histerectomía radical a través del abdomen. Cuando se extirpan los ganglios linfáticos, este procedimiento abdominal se llama histerectomía radical asistida por laparoscopía con linfa denectomía.

A veces se emplea también la cirugía laparoscópica asistida por robot para realizar las histerectomías radicales. En este método, el cirujano se sienta frente a un panel de control para mover con precisión los brazos robóticos que sostienen las herramientas quirúrgicas.

\section{La Cirugía Laparoscópica}

Resulta en menos dolor, menos pérdida de sangre durante la operación, y una hospitalización más corta en comparación con la cirugía abierta. Sin embargo, de acuerdo con la investigación reciente, las mujeres con cáncer de cuello uterino en etapas iniciales que se someten a una cirugía abierta suelen tener una menor probabilidad de que el cáncer regrese (recurra), así como una mejor probabilidad de vivir más tiempo que las mujeres que optan por una cirugía laparoscópica.

La cirugía laparoscópica puede ser todavía una opción para un pequeño grupo específico de mujeres con cáncer en etapa inicial, pero usted debe discutir detenidamente sus opciones con su médico.

Posibles efectos secundarios: Debido a que se extirpa el útero, esta cirugía resulta en infertilidad. Después de esta operación, algunas mujeres presentan problemas al vaciar sus vejigas porque se remueven algunos de los nervios que conducen a la vejiga. Puede que estas mujeres necesiten un catéter por un tiempo. Normalmente no hay complicaciones, pero pudiera presentarse sangrado excesivo, infección de la herida o daños a los sistemas urinario o intestinal. 


\section{Cuidados de las pacientes sometidas a cirugías de cáncer uterino}

Vol. 3, núm. 1., (2019)

María Belén Gárate Campoverde; Cástulo Leonel Moreira Moreira; Juan Carlos Zambrano Basurto; María José Saltos Loor; Stephane Paolo Guerrero Zambrano; Rómulo Argenis Muñoz

Moreira

La histerectomía radical no cambia la capacidad de una mujer de sentir placer sexual. Aunque se acorta la vagina, el área alrededor del clítoris y la membrana que recubre la vagina es tan sensitiva como antes. Una mujer no necesita un útero o cuello uterino para alcanzar un orgasmo. Cuando el cáncer ha causado dolor o sangrado con la relación sexual, la histerectomía puede en realidad mejorar la vida sexual de una mujer al eliminar estos síntomas. Para más información sobre cómo manejar los efectos secundarios sexuales del tratamiento del cáncer de cuello uterino.

\section{Cervicectomía.}

Otro procedimiento, conocido como cervicectomía radical, permite a las mujeres ser tratadas sin perder la capacidad de tener hijos. La operación se realiza a través de la vagina o el abdomen, y a veces se hace mediante laparoscopia (cirugía mínimamente invasiva). En este procedimiento se extirpa el cuello uterino y la parte superior de la vagina, pero no el cuerpo del útero. El cirujano coloca una sutura en "bolsa de tabaco" para que funcione como abertura artificial del cuello uterino dentro de la cavidad uterina. (Carett 2016)

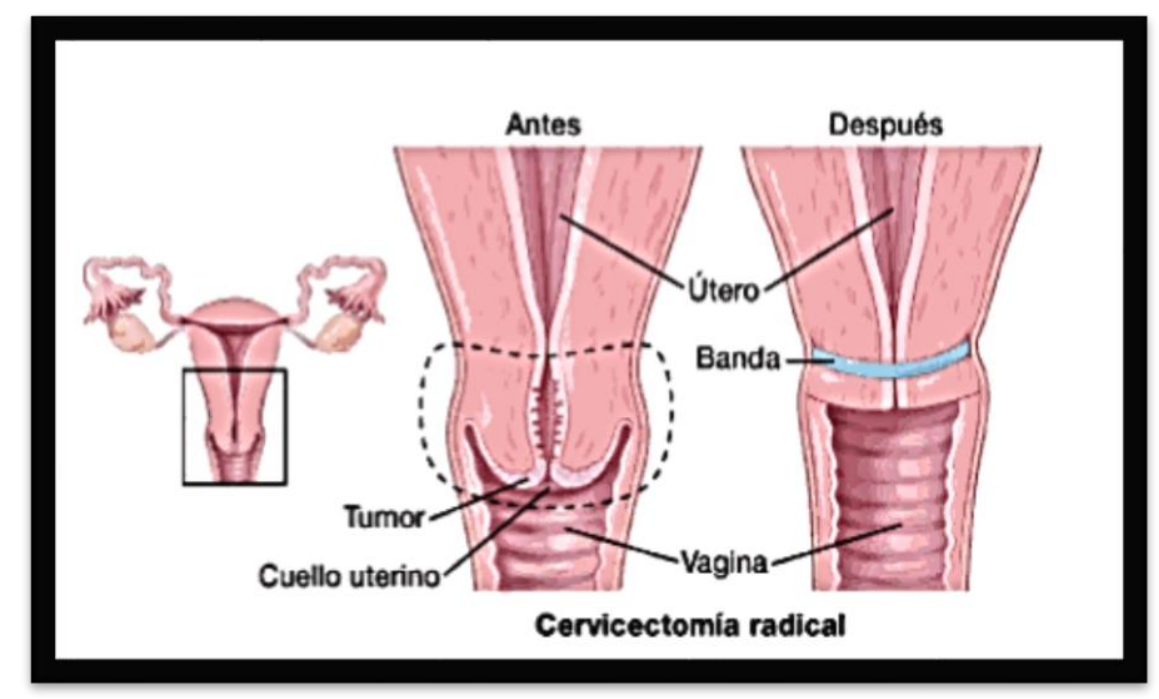

Fuente: (Carett 2016).

También se extirpan los ganglios linfáticos cercanos mediante una laparoscopia, lo que puede requerir otra incisión (corte). Esta operación se hace a través de la vagina o del abdomen. 


\section{Cuidados de las pacientes sometidas a cirugías de cáncer uterino}

Vol. 3, núm. 1., (2019)

María Belén Gárate Campoverde; Cástulo Leonel Moreira Moreira; Juan Carlos Zambrano

Basurto; María José Saltos Loor; Stephane Paolo Guerrero Zambrano; Rómulo Argenis Muñoz Moreira

Después de la cervicectomía, algunas mujeres pueden llevar a término un embarazo y dar a luz un bebé saludable por cesárea, aunque las mujeres que se han sometido a esta cirugía pueden tener un mayor riesgo de aborto espontáneo.

\section{Criocirugía:}

Se basa en una sonda de metal muy fría se coloca directamente en el cuello uterino. Esto destruye las células anormales mediante congelación. Esto se puede hacer en un consultorio médico o en una clínica. Después de la criocirugía, usted puede presentar mucha secreción acuosa de color café durante algunas semanas.

\section{Cirugía Láser:}

Se usa un rayo láser enfocado (a través de la vagina) para vaporizar (quemar) las células anormales o extirpar una porción pequeña de tejido para estudiarlo. Este procedimiento se puede realizar en el consultorio del médico o en la clínica usando un anestésico local (medicina que se usa para adormecer el área).

\section{Conización:}

Se extrae del cuello uterino un pedazo de tejido en forma de cono. Este procedimiento se hace utilizando un bisturí quirúrgico o bisturí láser (biopsia de cono con bisturí frío) o utilizando un alambre delgado calentado mediante electricidad (procedimiento electro quirúrgico, LEEP o LEETZ). Después del procedimiento, se examina el tejido extirpado con un microscopio. Si los márgenes (bordes más externos) del tejido contienen cáncer (o pre cáncer), lo que se conoce como márgenes positivos, es posible que algo de cáncer (o pre cáncer) haya quedado de modo que se necesita tratamiento adicional. 


\section{Cuidados de las pacientes sometidas a cirugías de cáncer uterino}

Vol. 3, núm. 1., (2019)

María Belén Gárate Campoverde; Cástulo Leonel Moreira Moreira; Juan Carlos Zambrano

Basurto; María José Saltos Loor; Stephane Paolo Guerrero Zambrano; Rómulo Argenis Muñoz

Moreira

\section{Exenteración Pélvica}

Esta operación es más extensa y puede emplearse para tratar el cáncer de cuello uterino recurrente. En esta cirugía, se extirpan todos los mismos órganos y tejidos que en la histerectomía radical con disección de los ganglios linfáticos pélvicos. (La disección de ganglios linfáticos se discute en la sección siguiente). Además, se pueden extirpar la vejiga, la vagina, el recto y parte del colon, dependiendo del lugar hacia donde se propagó el cáncer.

\section{Disección de los Ganglios Linfáticos Pélvicos}

El cáncer que se origina en el cuello uterino puede propagarse a los ganglios linfáticos (grupos de tejido del sistema inmunitario que tienen forma de fríjol) en la pelvis. Para saber si hay propagación a los ganglios linfáticos, el cirujano puede extirpar algunos de estos ganglios. Este procedimiento se conoce como disección de ganglios linfáticoso muestreo de ganglios linfáticos. Se realiza al mismo tiempo que se hace la histerectomía o la cervicectomía.

La extirpación de ganglios linfáticos puede derivar en problemas con el drenaje de líquido en las piernas. Esto puede causar hinchazón severa en las piernas, una condición llamada linfedema. Para obtener más información, consulte Linfedema.

\section{El Tratamiento Para Cáncer De Útero Avanzado Debe Comprender Los Siguientes Cuidados}

El tratamiento del cáncer de útero va a depender de la etapa de éste, del tamaño y forma del tumor, la edad, la salud general de la mujer y su deseo de tener hijos en el futuro Los tipos de cirugía para el cáncer cervical precoz comprenden:

Cono LEEP. - Procedimiento de escisión electro quirúrgica con asa, que utiliza electricidad para extirpar el tejido anormal. - Crioterapia. - que congela las células anormales.

Cirugía láser. - Terapia con láser que utiliza luz para cauterizar el tejido anormal. Histerectomía. - extirpación del útero, pero no de los ovarios a menudo no se lleva a cabo para el cáncer cervical que no se ha diseminado. 


\section{Cuidados de las pacientes sometidas a cirugías de cáncer uterino}

Vol. 3, núm. 1., (2019)

María Belén Gárate Campoverde; Cástulo Leonel Moreira Moreira; Juan Carlos Zambrano Basurto; María José Saltos Loor; Stephane Paolo Guerrero Zambrano; Rómulo Argenis Muñoz Moreira

\section{Tabla $\mathbf{N}^{\circ}$ 1. Extensión del Tumor de Cáncer Uterino}

\begin{tabular}{|c|c|}
\hline Clasificación & Descripión \\
\hline Tis & $\begin{array}{l}\text { Las células cancerosas sólo se encuentran en la superficie del } \\
\text { cuello uterino }\end{array}$ \\
\hline T1 & $\begin{array}{l}\text { Las células cancerosas han crecido desde la superficie del } \\
\text { cuello uterino hasta los tejidos más profundos del éste }\end{array}$ \\
\hline T1a & $\begin{array}{l}\text { Existe una cantidad muy pequeña de cáncer que sólo se puede } \\
\text { observar con un microscopio }\end{array}$ \\
\hline T1a1 & $\begin{array}{l}\text { El área de cáncer es de menos de } 3 \mathrm{~mm} \text { ( } 1 / 8 \text { de pulgada) de } \\
\text { profundidad y de menos de } 7 \mathrm{~mm}(1 / 4 \mathrm{de} \mathrm{pg}) \text { de ancho. }\end{array}$ \\
\hline T1a2 & $\begin{array}{l}\text { Área de invasión del cáncer es de entre } 3 \mathrm{~mm} \text { y } 5 \mathrm{~mm} \text { (1/5 de } \\
\mathrm{pg} \text { ) de profundidad y de menos de } 7 \mathrm{~mm}(1 / 4 \mathrm{de} \mathrm{pg}) \text { de ancho }\end{array}$ \\
\hline T1b & $\begin{array}{l}\text { Cánceres de Etapa I se pueden ver sin microscopio. Incluye los } \\
\text { tumores cancerosos que pueden ver con microscopio y se han } \\
\text { propagado más de } 5 \mathrm{~mm} \text { ( } 1 / 5 \mathrm{de} \mathrm{pg} \text { ) profundidad en el tejido } \\
\text { conectivo del cuello uterino o tienen más } 7 \mathrm{~mm} \text { de ancho }\end{array}$ \\
\hline T1b1 & $\begin{array}{l}\text { El cáncer se puede ver, pero no tiene más de } 4 \text { centímetros } \\
\text { (alrededor de } 13 / 5 \text { de pulgada). }\end{array}$ \\
\hline T1b2 & El cáncer se puede ver y tiene más de 4 centímetros \\
\hline
\end{tabular}

Fuente: (OMS 2016)

\section{Tratamiento}

Entre los múltiples tratamientos a realizarse en el tratamiento para el cáncer cervical más avanzado se logran conocer:

- Histerectomía radical. - Se extirpa el útero y mucho de los tejidos circundantes, incluyen los ganglios linfáticos y parte superior de la vagina.

- Evisceración pélvica. - Cirugía en la cual se extirpan todos los órganos de la pelvis, incluidos la vejiga y el recto.

- Radioterapia. - (Interna o Externa) Para tratarlos casos en que el cáncer se ha diseminado más allá de la pelvis o el cáncer que ha reaparecido. 


\section{Cuidados de las pacientes sometidas a cirugías de cáncer uterino}

Vol. 3, núm. 1., (2019)

María Belén Gárate Campoverde; Cástulo Leonel Moreira Moreira; Juan Carlos Zambrano

Basurto; María José Saltos Loor; Stephane Paolo Guerrero Zambrano; Rómulo Argenis Muñoz

Moreira

- Braquiterapia o radioterapia interna. - Se inserta un dispositivo lleno de material radiactivo dentro de la vagina de la mujer, cerca al cáncer cervical.

\section{Tabla $\mathbf{N}^{\circ}$ 2. Etapas del Cáncer de Cuello Uterino}

\begin{tabular}{|l|l|}
\hline Clasificación & \multicolumn{1}{c|}{ Descripión } \\
\hline T2 & $\begin{array}{l}\text { El cáncer crecio más allá del cuello uterino y el útero, pero no } \\
\text { se ha propagado a las paredes de la pelvis o la parte inferior de } \\
\text { la vagina. Puede haber crecido en la parte superior de la vagina }\end{array}$ \\
\hline T2a & $\begin{array}{l}\text { El cáncer no se ha propagado a los tejidos próximos al cuello } \\
\text { uterino (parametrio) }\end{array}$ \\
\hline T2a1 & El cáncer se puede ver, pero no tiene más de $4 \mathrm{~cm}$ (1 $3 / 5 \mathrm{de} \mathrm{pg)}$ \\
\hline T2a2 & El cáncer se puede ver y tiene más de 4 centímetros \\
\hline T2b & $\begin{array}{l}\text { El cáncer se ha propagado a los tejidos adyacentes al cuello } \\
\text { uterino (el parametrio) }\end{array}$ \\
\hline T3 & $\begin{array}{l}\text { El cáncer se ha propagado a la parte inferior de la vagina o a las } \\
\text { paredes pélvicas. El cáncer puede estar bloqueando los uréteres } \\
\text { (conductos que transportan la orina de los riñones a la vejiga) }\end{array}$ \\
\hline T3a & $\begin{array}{l}\text { El cáncer se ha propagado al tercio inferior de la vagina, pero no } \\
\text { a las paredes pélvicas }\end{array}$ \\
\hline T3b & $\begin{array}{l}\text { El cáncer ha crecido hacia las paredes de la pelvis y/o está } \\
\text { bloqueando uno o ambos uréteres (se le llama hidronefrosis). }\end{array}$ \\
\hline T4 & El cáncer se propagó a la vejiga o al recto, crece fuera de pelvis \\
\hline
\end{tabular}

Fuente: (OMS 2016).

Las mujeres que han recibido terapia combinada de cirugía y radioterapia, en comparación con las que sólo recibieron cirugía, se hacen activas sexualmente de forma más tardía. Por su parte, las mujeres que no fueron sometidas a cirugías reconstructivas, reportaron un deterioro en el funcionamiento sexual al presentar mayores impedimentos físicos y menor autoestima.

Ahora bien, si solo se habla de radioterapia, se puede decir que ésta es la que más altera el ámbito físico de la sexualidad, puesto que produce disfunción sexual persistente durante los dos 


\section{Cuidados de las pacientes sometidas a cirugías de cáncer uterino}

Vol. 3, núm. 1., (2019)

María Belén Gárate Campoverde; Cástulo Leonel Moreira Moreira; Juan Carlos Zambrano

Basurto; María José Saltos Loor; Stephane Paolo Guerrero Zambrano; Rómulo Argenis Muñoz Moreira

años posteriores al tratamiento, es decir, muchas tienen dificultad para completar el coito y a otras simplemente les fue imposible lograrlo.

En términos generales, el cáncer y su tratamiento producen síntomas y efectos secundarios físicos. También pueden causar efectos emocionales, sociales y financieros. El tratamiento de estos efectos se denomina cuidados paliativos o atención médica de apoyo. Los cuidados paliativos son una parte importante de la atención que se incluye junto con los tratamientos para retrasar, detener o curar el cáncer.

Se ha demostrado que los cuidados paliativos pueden mejorar la calidad de vida y ayudarlo a sentirse más satisfecho con el tratamiento que recibe. Puede comenzar a recibir cuidados paliativos apenas se entera de que tiene cáncer y continuar recibiendo este tipo de atención a lo largo del tratamiento y la recuperación. Por ejemplo, los sobrevivientes del cáncer que tienen síntomas o efectos secundarios nuevos o permanentes después de que han completado el tratamiento también pueden recibir cuidados paliativos.

Las personas que reciben el cuidado paliativo incluyen a la paciente y su familia. En los países en desarrollo el cuidado paliativo se brinda en el hogar de las pacientes debido a que el cuidado ofrecido en hospicios u hospitales puede ser caro, poco accesible, o poco viable. Adicionalmente, muchas mujeres eligen la opción de morir rodeadas por su propio entorno, en lugar de hacerlo en un lugar desconocido.

En lugar de enfocarse solamente en la atención médica, el cuidado paliativo también trata las preocupaciones no relacionadas directamente con la atención médica que tienen las mujeres cuando se acercan al final de sus vidas. La atención a las necesidades emocionales, sociales y espirituales de las personas puede ayudar a aliviar mucha de la tensión y soledad de una persona que se acerca a la muerte.

La comodidad básica de la persona enferma es muy importante. La mujer con una enfermedad terminal debe mantenerse limpia y se debe cambiar su posición en el lecho con frecuencia para 


\section{Cuidados de las pacientes sometidas a cirugías de cáncer uterino}

Vol. 3, núm. 1., (2019)

María Belén Gárate Campoverde; Cástulo Leonel Moreira Moreira; Juan Carlos Zambrano

Basurto; María José Saltos Loor; Stephane Paolo Guerrero Zambrano; Rómulo Argenis Muñoz

Moreira

prevenir problemas cutáneos y pulmonares. La nutrición también es importante por lo que debe brindarse líquidos en cantidad suficiente y alimentos apetitosos.

\section{Conclusiones.}

Las mujeres viven experiencias múltiples al verse enfrentadas al Cáncer de cuello Uterino CACU, y más aún al tratamiento oncológico que inevitablemente está ligado a ésta, siendo muchas veces lo más difícil de afrontar en el camino. La calidad de vida y experiencia adquirida en el proceso de tratamiento es totalmente subjetiva, y está fuertemente relacionada con el grado de satisfacción que tiene la persona con su estado físico, emocional, con su vida familiar, amorosa, y social, marcando fuertemente el sentido que desde ahí le atribuirá a su vida

Frente a esto, las habilidades de afrontamiento de las mujeres están condicionadas por la forma en que cada una acepte inicialmente el diagnóstico de cáncer, el tratamiento y los posibles efectos secundarios a los que se verá enfrentada; planteamiento éste de suma importancia considerándose que, entre las principales preocupaciones de las mujeres con CACU radica esencialmente el apoyo social que esperan recibir, el estrés general, el bienestar familiar (especialmente por los hijos), la vida sexual y de pareja, y los síntomas físicos asociados al tratamiento.

Finalmente, se encuentra lo relacionado con los cuidados paliativos, los cuales de manera general, son aplicados en pacientes con un mal pronóstico, es decir, con enfermedades en etapa terminal, este tipo de cuidados se basan específicamente en mejorar la calidad de vida de la paciente y ayudarla a sentirse más satisfecha con el tratamiento que recibe.

De igual manera, estas pacientes pueden comenzar a recibir cuidados paliativos apenas se entera de que tiene cáncer y continuar recibiendo este tipo de atención a lo largo del tratamiento y la recuperación. Por ejemplo, los sobrevivientes del cáncer que tienen síntomas o efectos secundarios nuevos o permanentes después de que han completado el tratamiento también pueden recibir cuidados paliativos 
Cuidados de las pacientes sometidas a cirugías de cáncer uterino

Vol. 3, núm. 1., (2019)

María Belén Gárate Campoverde; Cástulo Leonel Moreira Moreira; Juan Carlos Zambrano

Basurto; María José Saltos Loor; Stephane Paolo Guerrero Zambrano; Rómulo Argenis Muñoz Moreira

\section{Bibliografía.}

A., W. (1997). Tipos de Tratamiento de Cáncer de Cuello Uterino. Revista Médica y de obstetricia de imbiomed, 34.

Arias, F. (2010). Paradigmas de la Investigación Cientifica. España: Editorial: Luces.

Dávila, N. (2012). Paradigmas de la Investigación Cientifica. Pereire, Colombia: Editorial: Las Brisas.

Henalt, B. (2016). Tratamiento de Cáncer de útero. Revista Chilena de Onstetricia y Ginecología, 8.

Henalt, Y. (2016). Tipos de Cáncer de útero. Revista Mexicana de Oncología, 22.

OMS, O. M. (2017). Cirugías de Cáncer de Utero. Revista Salud Integral de la Universidad de la Rioja en Colombia, 12.

$$
\text { (c) (1) () (2) }
$$

RECONOCIMIENTO-NOCOMERCIAL-COMPARTIRIGUAL

CC BY-NC-SA

ESTA LICENCIA PERMITE A OTROS ENTREMEZCLAR, AJUSTAR Y CONSTRUIR A PARTIR DE SU OBRA CON FINES NO COMERCIALES, SIEMPRE Y CUANDO LE RECONOZCAN LA AUTORÍA Y SUS NUEVAS CREACIONES ESTÉN BAJO UNA LICENCIA CON LOS MISMOS TÉRMINOS. 